\title{
First hospitalization after kidney transplantation
}

\author{
Antonios H. Tzamaloukas
}

Received: 31 January 2012/ Accepted: 10 April 2012/Published online: 21 July 2012

(C) Springer-Verlag (outside the USA) 2012

Editor,

A 69-year old man with a kidney transplant was admitted for right inguinal hernia repair in September 2011. The surgery was successful, and he was discharged after a hospitalization that lasted less than $24 \mathrm{~h}$. He has been well since then. He developed end-stage renal disease at the beginning of 1972 and was placed on hemodialysis. He received from his brother a "perfect match" renal transplant in April 1973. The transplant has functioned very well on minimal doses of anti-rejection medications, azathioprin and prednisone. His latest serum creatinine level, in the beginning of 2012, was 1.07 $\mathrm{mg} / \mathrm{dL}$ (eGFR $69 \mathrm{~mL} / \mathrm{min}$ ), and urinalysis was normal.

He has had over the years several health issues including hypertension, prostatic hypertrophy, gastritis, irritable bowel and most importantly numerous excisions of basal cell carcinomas of the skin. However, the 2011 hospital stay of less than $24 \mathrm{~h}$ for hernia repair was his first hospitalization after the kidney transplantation.

For the past 33 years I have been following this remarkable man, who was told in 1973 that the life expectancy of his transplanted kidney, and probably himself, was at the most 15-20 years. I was given the opportunity to reflect on the factors that have led to survival with good quality of life for 40 years after the development

A. H. Tzamaloukas ( $\bowtie)$

Renal Section, Medicine Service, Raymond G. Murphy Veterans Affairs Medical Center, 1501 San Pedro, SE, Albuquerque, NM 87108, USA

e-mail: Antonios.Tzamaloukas@med.va.gov of end-stage renal disease. The perfect histocompatibility of the renal transplant is definitely a major reason for this long and successful survival. However, there is a second equally important reason. Throughout our long association, this transplant recipient has demonstrated great insight into all his health issues and a great initiative. His awareness of the actions and side effects of his medications is equal to or at times greater than that of health care professionals. He seeks medical attention as soon as he recognizes that something is wrong. On his own, he will study diligently about the treatment options of his ailments and will discuss these options in detail with his physicians. He chooses the treatment after careful consideration. Invariably, treatment takes place early in the course of disease. For example, it was he who discovered his skin lesions. His timely finding and appreciation of the significance of these lesions led to cure. He did not learn this behavior from me or my predecessors. On the contrary, I learned from him to respond to his telephone calls with haste. To complete the picture, the kidney donor is alive and without medical issues.

I want to share with the readers of the journal the lesson that this patient gave me: The long-term success of medical care for complex medical conditions requiring close follow-up and many medications with potentially serious side effects relies heavily on the level of knowledge and the active involvement of the patient in his/her care. The challenge for health care professionals is to instill this idea into the minds of patients who do not have naturally the mental makeup to be the responsible persons for their care. 